\title{
TEORIA E PRÁTICA: CULTURA MAKER E EXPERIÊNCIA DE INICIAÇÃO CIENTÍFICA VOLUNTÁRIA
}

\author{
THEORY AND PRACTICE: MAKER CULTURE AND \\ VOLUNTARY SCIENTIFIC INITIATION EXPERIENCE
}

Henrique Alves Camargo ${ }^{1}$

Carina Merkle Lingnau ${ }^{2}$

\begin{abstract}
RESUMO
O objetivo desta pesquisa é relacionar a cultura maker como um dispositivo foucaultiano baseado na experiência prática com a empresa Edukamaker e com o embasamento teórico de Foucault. Como metodologia buscamos o referencial bibliográfico, vídeos e materiais coletados nas atividades práticas. Como resultados percebemos a cultura maker como dispositivo que aciona o saber em sujeitos que não percebiam em si mesmos o potencial do conhecimento e que por vezes eram invisibilizados pela sociedade do conhecimento.

Palavras-chave: Discurso. Educação. Foucault.
\end{abstract}

\section{ABSTRACT}

The maker culture is a creative practice for dealing with knowledge and problem solving. We relate the maker culture and the Foucaultian device. As methodology we seek the bibliographic reference, videos and materials collected in practical activities. As a result, we perceive the maker culture as a device that triggers knowledge in subjects who did not perceive the potential of knowledge in themselves and who were sometimes made invisible by the knowledge society.

Keywords: Discourse. Education. Foucault.

\footnotetext{
1 Graduando em Engenharia de Software na UTFPR. Sócio na empresa startup voltada à educação: Edukamaker. E-mail: hcamargo@ alunos.utfpr.edu.br. Endereço para acessar este CV: http://lattes.cnpq.br/7483218298261234. ORCID id: https://orcid.org/0000-0001-8802-0839.

2 Doutora em Letras. Mestra em Educação. Graduada em Letras. Professora em cursos de graduação da UTFPR-FB e no curso de pós-graduação, Mestrado em Educação, da Universidade Estadual do Oeste do Paraná, campus Francisco Beltrão (UNIOESTE-FB). E-mail: carinalingnau@utfpr.edu.br. Endereço para acessar este CV: http://lattes.cnpq.br/2891086165049465. ORCID id: https://orcid.org/0000-0002-8469-3961.
} 


\section{INTRODUÇÃO}

O registro desse artigo envolve a pesquisa e a prática das atividades da empresa Edukamaker cuja propriedade está sob os cuidados do acadêmico, primeiro autor deste artigo, como sendo um dos sócios do negócio que nasceu na incubadora da Universidade Tecnológica Federal do Paraná, campus Francisco Beltrão (UTFPR-FB).

A empresa Edukamaker é uma startup que nasceu entre acadêmicos no Hotel Tecnológico da UTFPR-FB, um espaço que busca ajudar projetos de criação de empresas, para tanto passam por processos seletivos, orientação e ingresso em suas empreitadas. Assim nasceu a Edukamaker, empresa que tem se destacado no ramo de educação aliada à tecnologia que envolve a execução de projetos junto com os alunos. Como discente do Curso de Licenciatura em Informática na UTFPR-FB, inserido no projeto de pesquisa $O$ dispositivo das metodologias ativas: reflexões e apontamentos o discente teve como plano de trabalho desenvolvido para esta pesquisa: Cultura maker como dispositivo foucaultiano.

$\mathrm{O}$ objetivo desta pesquisa então é relacionar a cultura maker como um dispositivo foucaultiano baseado na experiência prática com a empresa Edukamaker e com o embasamento teórico de Foucault (2008, 2014). E Para organizar este artigo dividimos o texto nas seguintes partes: metodologia, cultura maker, desenvolvendo o projeto prático, Foucault e o dispositivo, resultados e discussão, referências.

Este trabalho foi bibliográfico, qualitativo e empírico (BAUER E GASKEL, 2002). O processo da pesquisa realizada teve início como discente pesquisador voluntário, após os estudos dos textos e vídeos sugeridos, para compreender Michel Foucault (2008, 2014). Junto às análises deu-se início as aulas que seriam objeto de estudo aplicadas “in loco”, abordando a temática cultura maker (DOUGHERTY, 2012a).

A metodologia para a pesquisa empírica teve como recorte ações de cunho educativo da empresa Edukamaker, o que foi desenvolvido da seguinte forma: as aulas foram aplicadas semanalmente às quartas e quintas-feiras em uma das escolas pública de ensino integral do município de Francisco Beltrão, abrangendo cerca de aproximadamente 100 alunos de idade entre 9 a 11 anos divididos em quatro turmas.

\section{Cultura maker}

O que é cultura maker? Cultura maker nada mais é que um movimento, que se caracteriza pela ideia de colocar a mão na massa, encontrar soluções criativas para resolver problemas, criar objetos e equipamentos por meio do fazer, da experimentação, do erro, ou seja, é a prática aplicada caracterizada por meios digitais ou não, com auxílio de ferramentas e equipamentos tradicionais ou não (DOUGHERTY, 2012a). 
Destacando que o makerspace nada mais é do que um espaço ou sala, normalmente comunitários, usados como oficinas de criação, muitas vezes equipados de ferramentas e materiais que auxiliam na construção de projetos, produtos e serviços Em seguida apresentamos as Figuras 01 e 02 imagem está no site da Edukamaker e mostra o robô pronto à esquerda e em montagem à direita (DOUGHERTY, 2012a).

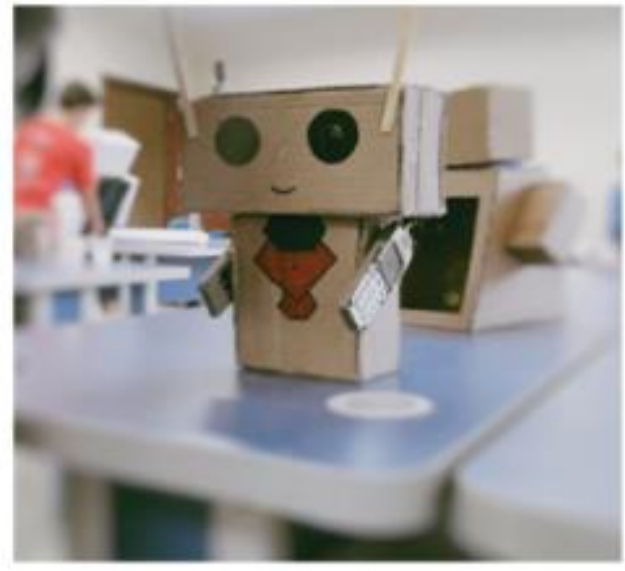

Figura 01 - Cultura maker

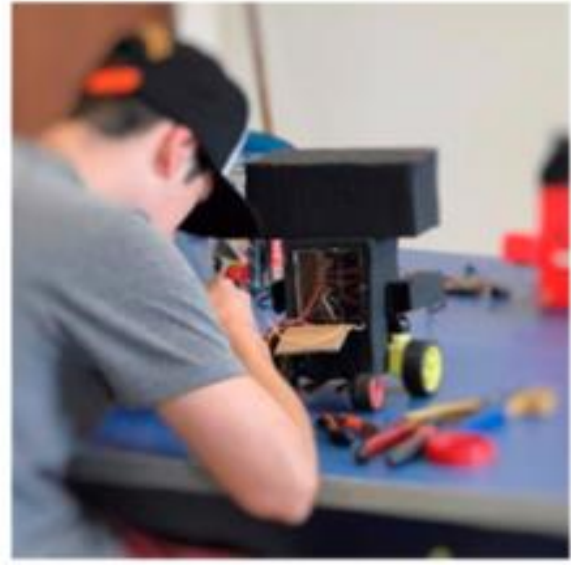

Figura 02: Makerspace Fonte: http://edukamaker.com.br/. Acessado em: 30/09/2020.

No detalhamento das figuras acima podemos observar que a figura 01 representa um projeto realizado dentro do curso de Gambi Art Tech, neste curso os alunos aprendem a desenvolver projetos voltados as artes, gambiarras artísticas que auxiliam, fomentam e desenvolvem o conhecimento e conceitos de técnicas artísticas, como pintura, esculturas, dobraduras, mas atreladas as áreas técnicas como eletrônica, programação e automação. Na figura o aluno usou o papelão, palitos, tintas e cola quente para desenvolver um robô e usou alguns aparelhos celulares como braços uma vez que não conseguiu hackear os aparelhos para dar outro uso. Já a figura 2 apresenta um aluno desenvolvendo a parte eletrônica de seu projeto/robô, controle e automação, lógica e programação são áreas desenvolvidas dentro do curso de robótica para execução e entrega nos projetos. A importância dessa prática é trabalhar com a base de experiência e conhecimento dos alunos, executar na prática tudo que ele já desenvolveu de forma teórica para poder montar e validar, ver funcionar esse primeiro protótipo.

Assim, verificamos que aplicando o projeto maker e identificando oportunidades voltadas à temática maker em escolas é um processo simples, porém deve se ater a diversos cuidados, tais como: objetivos a curto e longo prazo; divisão de grupos; escolha de equipamentos e materiais; sensibilização para o conhecimento; validação dos projetos. 


\section{Desenvolvendo o projeto prático}

Para guiar os alunos durante a execução prática adotou-se um infográfico (FIGURA 03), sendo uma referência de caminho ou jornada que planificava os esforços e dava visibilidade aos projetos que foram trabalhados, cabendo ao professor e alunos indicarem em quais etapas cada projeto estava e para qual deveria prosseguir.

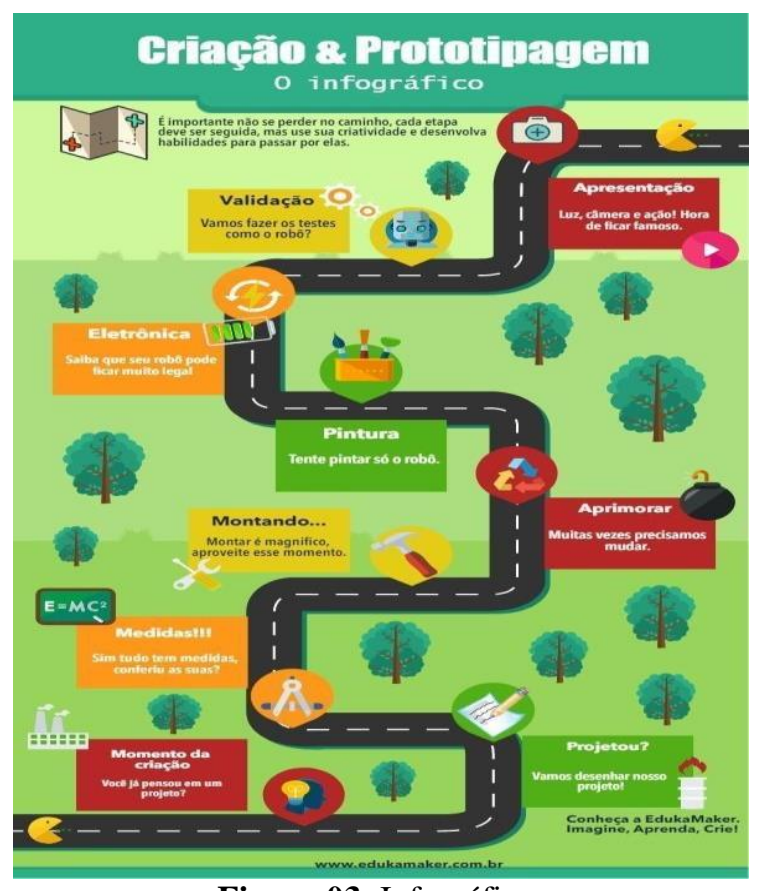

Figura 03: Infográfico.

Fonte: http://edukamaker.com.br/. Acessado em: 30/09/2020

O Infográfico, disposto pela Figura 03, foi desenvolvido pela Edukamaker e representa o caminho a ser percorrido desde a ideia, passando pelas etapas até a concreta execução do projeto. Ao percorrer o infográfico foi preciso atentar para os seguintes pontos:

Metas: para ajudar a identificar melhor as oportunidades e quais projetos pode-se trabalhar durante o curso e que melhor agrade os alunos, uma enquete foi proposta: "O que você pretende ser quando crescer?”, para análise dos resultados desta primeira enquete, os discentes foram divididos em grupos conforme resposta dos alunos e suas escolhas, então temos:

Bem-estar e Saúde: esta categoria engloba profissões e áreas como medicina, fisioterapia, zoologia, veterinária, massagista, cabeleireira, esteticista, nutricionista, ao todo no início do curso 22 alunos escolheram.

Educação, Tecnologia e Engenharia: abrange profissões como pedagogia, matemática, engenharias, jogos, arquitetura, ao todo no início do curso 12 alunos escolheram.

Mobilidade e Transporte: aborda áreas como motorista, logística, carregador, manobrista, ao todo no início do curso - 11 alunos. 
Segurança: aborda polícia civil, polícia federal, polícia militar, militar, segurança, ao todo no início do curso - 7 alunos.

Artes: aborda áreas como cinema, ator e atriz, apresentador de TV, cantor, teatro, ao todo no início do curso - 4 alunos.

Divisão de grupos: A divisão de grupos se dá por meio das afinidades, cabe ao professor criar os meios ideais para criação destes grupos, o formato de trabalho a ser aplicado pode ou não intensificar atritos entre os grupos e de forma alguma deve se estabelecer algum tipo de competição entre estes grupos.

Escolha de equipamentos e materiais: a escolha de materiais e equipamentos a serem usados dentro dos espaços makers também é determinante para o sucesso dos projetos propostos. Neste processo deve se pensar qual a quantidade certa dos equipamentos, para facilitar o senso de partilha deve-se colocar um número menor do que a quantidade de alunos, mas não inferior à quantidade de grupos propostos, pois assim os alunos irão sempre compartilhar tais ferramentas. Já os materiais, devem ser abundantes, porém de forma a não ser perceptível diretamente pelos alunos, pois assim o uso se torna mais racional e sem desperdícios.

Sensibilização: o maker é um espaço multidisciplinar que sempre manterá relações com diversas áreas de conhecimento, como a matemática, português, inglês, física, mecânica, química, robótica, engenharias, gamificação e cabe ao professor fazer a "ponte" entre estas áreas para então o aluno criar tais relações e ampliar seu conhecimento e aspirações.

Validação: validar é muito mais que somente testar, apesar de ter o momento de testar o projeto, o ato de validar também é o de pertencimento, a relação do aluno para com o projeto, que confirma o protagonismo do aluno, "Fui eu que fiz!" "Esse é meu robô", estas são algumas falas que confirmam a validação do aprendizado. Para uma questão de registro, a validação pode ser feita por vários meios. Aqui foi realizado nova enquete, podendo analisar qual foi a percepção por parte dos alunos diante do desenvolvimento de novas habilidades no curso e obteve-se como resultado mais alunos envolvidos em questões relacionadas à educação, engenharia e tecnologia.

Outros: esta categoria englobava escolhas tais como mendigo, empresário, vendedor, jogador de futebol, ao todo no início do curso - 7 alunos.

Desse modo, pôde-se verificar aspectos da atividade prática da pesquisa que determinaram as relações entre o dispositivo foucaultiano e a cultura maker analisadas neste trabalho. Pois, quando discutimos sobre dispositivo e tecnologia muitas vezes relacionamos o vocábulo dispositivo apenas com aparelhos, peças, máquinas. Mas no caso deste artigo, 
relacionamos o dispositivo ao conceito trabalhado por Foucault (2008, p.244) que compreende o dispositivo como:

[...] um conjunto decididamente heterogêneo que engloba discursos, instituições, organizações arquitetônicas, decisões regulamentares, leis, medidas administrativas, enunciados científicos, proposições filosóficas, morais, filantrópicas. Em suma, o dito e o não dito são os elementos do dispositivo. O dispositivo é a rede que se pode estabelecer entre estes elementos.

Assim, no caso do dispositivo pensado por Foucault (2008) verificamos que a cultura maker funciona como esse dispositivo por reunir um conjunto de ações e teorias que impulsionam o modelo educacional para um movimento diferente do convencional, o qual se conecta em uma rede própria de pensar a educação como algo libertador, compartilhado e que permite a realização de alguma coisa concreta, funcional e que resolve problemas.

\section{Dispositivos \& o Movimento Maker}

Esse trabalho opera com o termo "dispositivo" justamente como uma forma de responder a uma certa urgência, neste caso, o desenvolvimento do saber em rede, enquanto movimento educacional, torná-lo mais dinâmico, criando uma releitura do conhecimento já adquirido pelo aluno e trazendo ao mesmo novos conhecimentos e novas formas de obter outras habilidades, tornando sua educação algo mais rico e significativo, mas compreendendo as limitações únicas de cada indivíduo (FOUCAULT, 2008).

Identificar as "linhas" do conhecimento, mapeá-las junto aos alunos, transforma a educação e seu aprendizado algo mais rico, porém mesmo tendo um "norte" mostra que existem várias maneiras e caminhos para chegar ao objetivo final, e cada aluno deve então identificar, entender e compreender, utilizando também de várias ferramentas para seu aprendizado que por mais que seja personalizado é usado do coletivismo para criar as relações do ser com o mundo. Como evidenciado nas figuras 04 e 05 , do que foi realizado com os alunos.

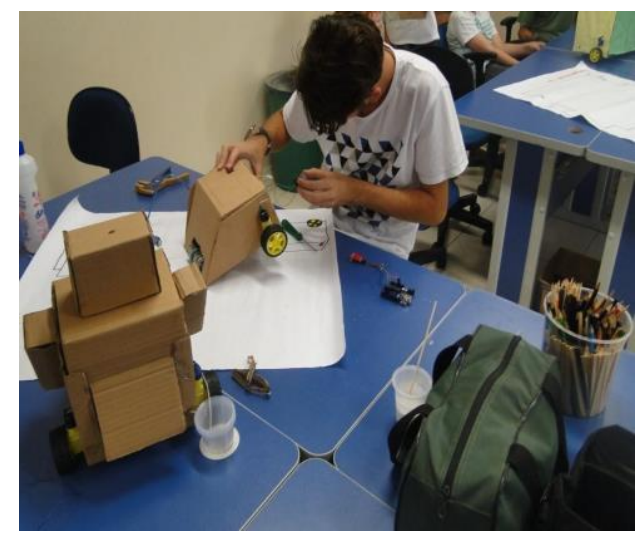

Figura 04: Aprendendo a criar.

Fonte: http://edukamaker.com.br/. Acessado em: 30/09/2020. 
A figura 04 mostra o aluno desenvolvendo seu projeto eletrônico com referência em um projeto confeccionado por ele, como mostra a folha branca sobre a mesa em que o aluno tem o projeto desenhado. O estudante utiliza um projeto de um outro robô que está a sua frente para verificar as conexões, ligações e equipamentos. Neste momento, ele avalia qual é a melhor forma de garantir as mínimas funcionalidades do seu projeto para que ele possa vir a executar movimentos mínimos necessários para validar. O colaborativismo entre equipes e alunos é uma forma de garantir que o conhecimento seja replicado, experiências tenham maior impacto no aprendizado, projetos em equipes e depois individualizados garantem uma maior segurança e qualidade entre os próprios alunos e entre professor-aluno.

Já a figura 05 apesenta a criação de um projeto menos sofisticado, porém não menos simples, uma vez que cada aluno tem uma percepção única e autêntica de pertencimento ou execução de seus projetos. Em sua montagem o aluno está trocando alguns módulos junto com o Arduino, pois devido à problemas técnicos na validação do seu protótipo, que também é chamado de MVP (do inglês Mínimo Produto Viável), não pode executar as devidas funcionalidades as quais foram programadas para exercer. A substituição dos componentes, uso de ferramentas para tais trocas, bem como os usos de equipamentos eletrônicos dentro do espaço maker é algo livre para os alunos, ampliando o sentimento de segurança e pertencimento do espaço.

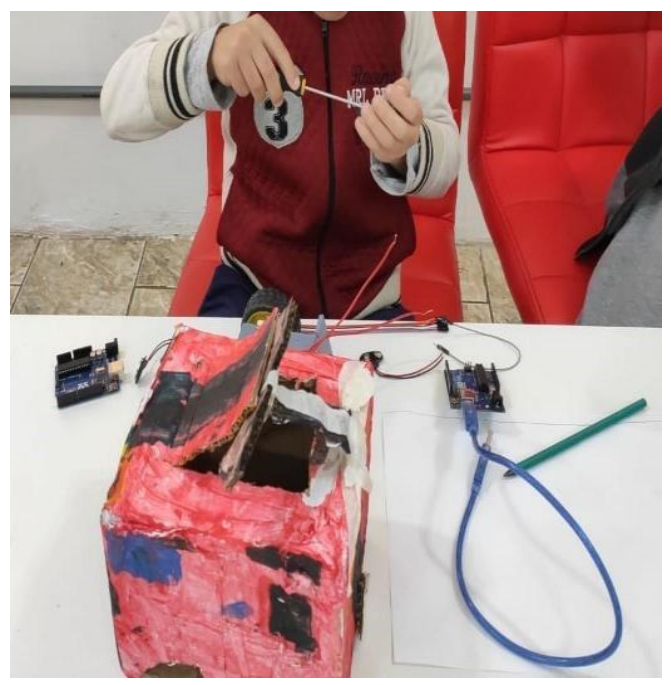

Figura 05: Montagem.

Fonte: http://edukamaker.com.br/. Acessado em: 30/09/2020.

Como dispositivo ainda podemos levar em conta a representatividade da "visibilidade e invisibilidade" uma vez que estes alunos diante de um universo educacional não possuem "visibilidade" e são vistos muitas vezes como números, ou seja, são "invisíveis". No entanto, dentro do "maker space", ganham visibilidade, personalizam seu aprendizado, rompem barreiras e limites que antes eram até impostos, para conseguirem validar seus projetos. 
Dougherty (2012b, p.1, tradução nossa) afirma que:

O movimento maker tem a oportunidade de transformar a educação, convidando os alunos a serem algo diferente dos consumidores da educação. Eles podem se tornar fabricantes e criadores de suas próprias vidas educacionais, passando de serem direcionados a fazer algo para se tornarem aprendizes autônomos e independentes. Cada vez mais, eles podem tirar proveito de novas ferramentas de expressão criativa e para explorar o mundo real ao seu redor. Eles podem ser participantes ativos na construção de um novo tipo de educação para o século 21.

Afinal, a aplicação da cultura maker, além de favorecer o protagonismo e desenvolver novas habilidades, modifica as relações do aluno com o mundo, o torna mais promissor para a conquista de seu espaço, o prepara para novos desafios e contribui para o enriquecimento sócio educacional.

\section{Considerações Finais}

A partir dessa experiência teórica com a bolsa de iniciação científica voluntária e da experiência prática com a startup Edukamaker foi possível avaliar a relação entre o dispositivo foucaultiano e as práticas educacionais do Movimento Maker incorporadas e aplicadas através da Edukamaker. A experiência mostrou que o dispositivo Movimento Maker ao ser percebido nas atividades educacionais apresenta efeitos de autonomia, cooperativismo e capacidade de resolver problemas. Assim, é possível identificar o dispositivo do Movimento Maker como uma perspectiva de ensino-aprendizagem que não só disponibiliza o conhecimento teórico, mas se preocupa com as relações de trocas entre os envolvidos estimulando ao mesmo tempo o crescimento individual e coletivo.

\section{Referências}

BAUER, M. W; GASKEL, G. Pesquisa qualitativa com texto, imagem e som: um manual prático I tradução de Pedrinho A. Guareschi. Petrópolis, RJ: Vozes, 2002.

DOUGHERTY, D. The maker movement, 2012a. Disponível em: $<<$ https://www.mitpressjournals.org/doi/pdf/10.1162/INOV_a_00135>>. Acesso: $11 \mathrm{mar}$. 2020 .

DOUGHERTY, D. Learning by making: American kids should be building rockets and robots, not taking standardized tests, 2012b. Disponível em: $<<$ https://slate.com/technology/2012/06/maker-faire-and-science-education-american-kidsshould-be-building-rockets-and-robots-not-taking-standardized-tests.html $>$. Acesso em: 30 set. 2020.

EDUKAMAKER. Somos uma startup de robótica educacional. Disponível em: $<<$ http://edukamaker.com.br/quem-somos/>>. Acesso em: 30 set. 2020. 
EDUKAMAKER. A Edukamaker, a cultura maker solucionando problemas! Disponível em: $\langle<$ https://www.instagram.com/edukamaker/>>. Acesso em: 30 set. 2020.

FOUCAULT, M. Microfísica do poder. Org e trad. Roberto Machado. 25a.ed. Rio de Janeiro: Edições Graal, 2008.

FOUCAULT, M. A arqueologia do saber: Tradução Luiz Felipe Baeta Neves. $8^{\text {a }}$.ed. Rio de Janeiro: Forense Universitária, 2014.

Artigo recebido em: 21/12/2020. Artigo aceito em: 24/01/2021. 